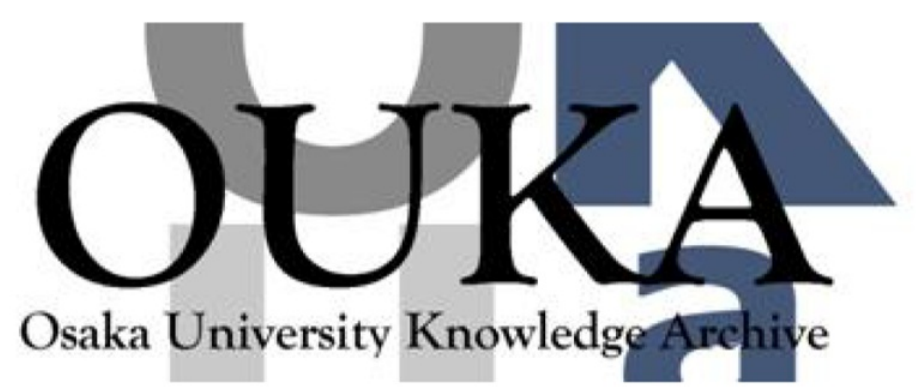

\begin{tabular}{|c|l|}
\hline Title & Electro-tunable liquid crystal waveguide laser \\
\hline Author(s) & $\begin{array}{l}\text { Matsui, Tatsunosuke; Ozaki, Masanori; Yoshino, } \\
\text { Katsumi }\end{array}$ \\
\hline Citation & $\begin{array}{l}\text { Proceedings of SPIE - The International Society } \\
\text { for Optical Eng ineering. 5518 p.220-p. 231 }\end{array}$ \\
\hline Issue Date & $2004-10-15$ \\
\hline oaire:version & VoR \\
\hline URL & https://hdl. handle. net/11094/76949 \\
\hline rights & \\
\hline Note & \\
\hline
\end{tabular}

Osaka University Knowledge Archive : OUKA

https://ir. Library. osaka-u. ac. jp/

Osaka University 


\title{
Electro-tunable liquid crystal waveguide laser
}

\author{
Tatsunosuke Matsui, Masanori Ozaki and Katsumi Yoshino \\ Department of Electronic Engineering, Graduate School of Engineering, Osaka University \\ 2-1 Yamada-Oka, Suita, Osaka 565-0871, JAPAN
}

\begin{abstract}
Electrically tunable laser action has been demonstrated in a dye-doped nematic liquid crystal (NLC) waveguide by holographic excitation. The optical feedback were provided by a transient grating induced by two-beam interference using Lloyd mirror configuration, and the distributed feedback (DFB) laser action was observed. Electrical tuning of lasing wavelength was realized due to the change of the effective refractive index of the NLC core layer caused by the reorientation of NLC molecules. The total shift of lasing wavelength was about $30 \mathrm{~nm}$, which could be realized with less than about $1.4 \mathrm{~V}$ of applied voltage. Based on a waveguiding mode theory, numerical analysis of TM-guided mode in the presence of applied electric field was performed, and field-induced tuning of the lasing wavelength was investigated in detail. Prospects for the realization of a single-mode operation and tuning of the lasing wavelength was also shown. Based on the numerical results, single-mode operation of lasing was experimentally realized utilizing NLC with low refractive indices.
\end{abstract}

Keywords: liquid crystal, tunable laser, distributed feedback laser, waveguide, holographic excitation

\section{INTRODUCTION}

From the first demonstration of a distributed feedback (DFB) lasing by Kogelnik et al., ${ }^{1}$ many studies on the DFB lasing have been reported. ${ }^{2}$ They demonstrated a laser action upon photoexcitation of a rhodamine 6G doped gelatin film with holographically inscribed phase grating (Fig.1 (a)). In their device, the optical feedback was provided by a backward Bragg scattering from phase grating, and mirrorless DFB laser action was observed. Lasing based on the DFB is available for organic and polymeric materials in which cleaved mirror cannot be obtained.

Besides their wide selectivity of emission wavelength range, laser device utilizing organic material has many advantages to the inorganic one such as self-organizability and flexibility. Recently, optically pumped DFB laser

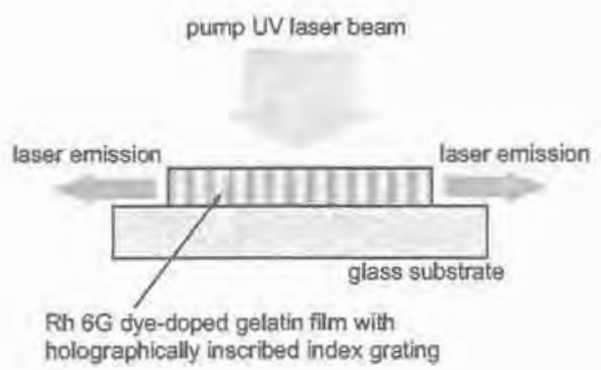

(a)

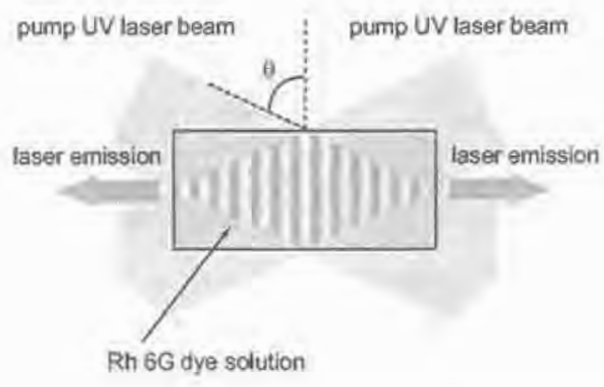

(b)

Figure 1. Schematic representation of pioneer studies on DFB laser actions reported in 1971. (a) laser action upon photoexcitation of a Rhodamine $6 \mathrm{G}$ doped gelatin film with holographically inscribed phase grating. ${ }^{1}$ (b) laser action by pumping a Rhodamine 6G dissolved in methanol-benzyl alcohol mixture with the fringes formed by the interference of two laser beams. ${ }^{15}$

Send correspondence to M.O.: E-mail: ozaki@ele.eng.osaka-u.ac.jp 


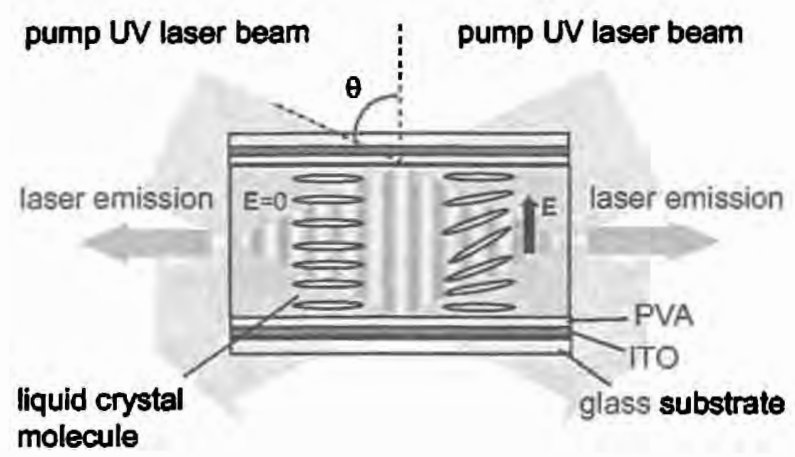

Figure 2. Schematic representation of the cell structure used in this study for the electrical tuning of the laser emission upon holographic excitation.

action has been observed using cholesteric liquid crystal $(\mathrm{LC})^{3-7}$ and ferroelectric $\mathrm{LC}^{8,9}$ as a self-organized onedimensional photonic crystal (PC). Moreover, polymeric flexible DFB lasers were fabricated using polymeric cholesteric LC, ${ }^{5,7}$ and surface relief grating structure fabricated by a micro-molding technique ${ }^{10}$ and more easily by using photochromic azo-polymer as a template. ${ }^{11}$

Another feature of organic materials is their tunability. Organic materials are very sensitive to external fields such as electric fields, magnetic fields, light, and so on. Using such sensitivities of organic materials, optical properties of materials such as refractive index can be tuned. Among various organic materials, LC is a favorable candidate for electrical tuning devices. LC molecules have various anisotropic properties such as optical, dielectric and magnetic anisotropies, which are caused by their elongated rod-like shapes and their directional order. Optical anisotropy of LC molecules results in an appearance of two refractive indices, that is, an extraordinary refractive index $n_{e}$ along the molecular long axis (director) and an ordinary refractive index $n_{o}$ perpendicular to the director. Besides it, dielectric anisotropy of LC molecules enables an electric field-induced reorientation of LC molecules. ${ }^{12}$ This indicates that, if LC is used as an active material for a laser action, an effective refractive index of the laser medium $n_{e f f}$ can be electrically controlled due to the field-induced reorientation of LC molecules, and various studies have been reported employing LC molecules into micro-cavity structures such as one dimensional PC composed by dielectric distributed Bragg reflector (DBR), ${ }^{13}$ and so on.

In this article, we demonstrated an electro-tuning of DFB laser emission using a dye-doped nematic LC (NLC) waveguide as an active medium by a transient grating using interference fringes induced by two excitation laser beams (holographic excitation). ${ }^{14}$ In this work, a planarly aligned uniform cell was fabricated and an electrotuning of laser emission was investigated using the electric field-induced spray-bend deformation of the LC molecules (Fig.2). DFB lasing based on the holographic excitation was demonstrated first by Shank et.al. ${ }^{15}$ They observed the DFB lasing by pumping a Rhodamine 6G dissolved in methanol-benzyl alcohol mixture with the fringes formed by the interference of two laser beams (Fig.1 (b)). The wavelength of laser emission $\lambda_{B \text { ragg }}$ upon holographic excitation can be expressed by a following equation,

$$
\lambda_{\text {Bragg }}=\frac{n_{e f f} \lambda_{e x}}{m \sin \theta}
$$

where $n_{e f f}$ is the effective refractive index of the active medium, $\lambda_{e x}$ is the wavelength of excitation beams, $m$ is the order of diffraction and $\theta$ is the half angle between two excitation beams. Based on this equation, a tunability of the laser emission wavelength was also demonstrated by changing the angle between two excitation beams $2 \theta$ or the refractive index of the laser dye solution $n_{e f f}$. In our case, tuning of lasing wavelength was realized by tuning $n_{\text {eff }}$ using the electric-field-induced reorientation of NLC molecules. In order to explain the experimental results in detail, numerical analyses of guided modes under applied voltages were also performed. Moreover, single-mode operation of this laser are also demonstrated. 


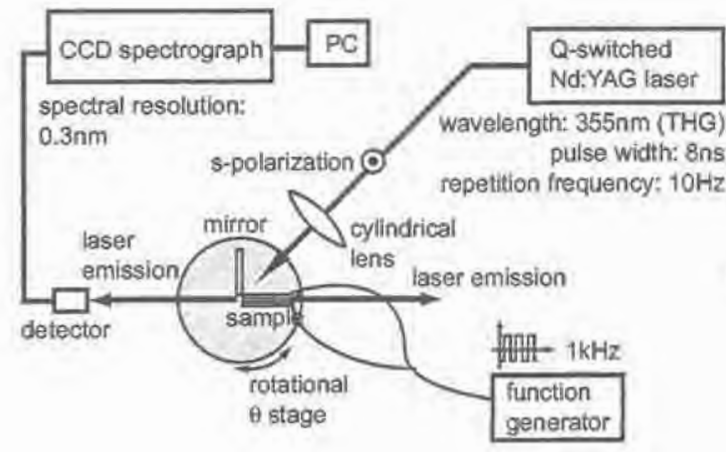

(a)

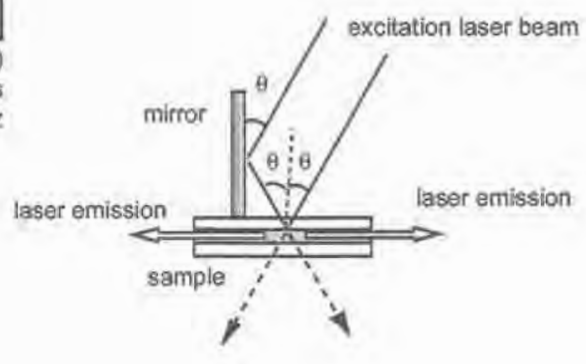

(b)

Figure 3. (a) Schematic representation of the experimental setup for emission measurement. (b) the Lloyd mirror setup for the interferential illumination.

\section{EXPERIMENTAL AND NUMERICAL ANALYSIS}

\subsection{Materials and device structure}

The NLC mixture E-44 (Merck) was used as a host material. The dielectric anisotropy of this NLC is positive so that if an electric field is applied to LC layer, LC molecules align their molecular long axis along the applied electric field as shown in Fig.2. As a laser dye dopant in the NLC, [2-[2-4-(Dimethylamino)phenyl]ethenyl]-6methyl-4H-pyran-4-ylidene propanedinitrile, DCM (Exciton) was used. The concentration of the dye was 0.7 wt.-\%. Ordinary and extraordinary refractive indices $n_{0}$ and $n_{e}$ of this mixture are 1.53 and 1.78 , respectively (at $632.8 \mathrm{~nm}$ ).

A cell structure used in this study is schematically shown in Fig.2. A sandwiched cell was composed of two Indium-Tin-oxide (ITO)-coated quartz glass substrates. The cell gap was $2.5 \mu \mathrm{m}$. $\mathrm{SiO}_{2}$ microspheres were used as spacers. The surfaces of substrates were coated with a poly (vinyl alcohol) (PVA) and rubbed in order to obtain a planarly aligned cell in which the direction of LC initially aligns parallel to the substrates. The sample was inserted in the isotropic phase by a capillary action. In such a cell structure, the PVA layer also acted as a low index clad layer because refractive index of PVA layer $n_{c}\left(=n_{P V A}=1.52\right)$ was lower than $n_{o}$ of NLC. On the other hand, the NLC layer acted as a core layer of the slab waveguide and an electromagnetic wave can be confined effectively in the NLC layer under the total reflection condition. ${ }^{16,17}$

\subsection{Experimental setup of optical measurement}

Figure 3 (a) shows an experimental setup for emission measurement. A third harmonic light of Q-switched Nd:YAG laser (Spectra Physics, Quanta-Ray INDI) was used for excitation, whose wavelength, pulse width and pulse repetition frequency were $355 \mathrm{~nm}, 8 \mathrm{~ns}$ and $10 \mathrm{~Hz}$, respectively. The polarization of both two excitation laser beams was set to be s-polarization, that is, perpendicular to the incident plane, in order to make photoinduced transient grating to be a pure intensity grating. ${ }^{18,19}$ The excitation laser beams were focused into a stripe using a cylindrical lens. The $\mathrm{Al}$ mirror was placed in front of the sample cell perpendicular to the cell, so that so-called Lloyd mirror setup for the holographic illumination was realized as shown in Fig.3 (b). The sample cell and the mirror were fixed on a rotational stage, and the angle between two excitation laser beams $2 \theta$ was adjusted by the rotational angle of this stage. In this configuration, the rubbing direction in the sample cell was perpendicular to the rotational axis of the stage. That is, the director of NLC molecules in the cell lies in the incident plane of two excitation beams. Under such an illumination condition, both transverse electric (TE) and transverse magnetic (TM) -guided modes in the NLC core layer feel the ordinary refractive index $n_{0}$ of the NLC in the absence of the electric field. The emission spectra from the sample were measured utilizing a spectrograph with a charge-coupled device (CCD) detector (Oriel, Multi Spec 257) having a spectral resolution of $0.3 \mathrm{~nm}$. For the application of an electric field to the sample cell, a function generator (Hewlett Packard, 3314A) was used. A rectangular shaped $\mathrm{AC}$ voltage of $1 \mathrm{kHz}$ was applied. 


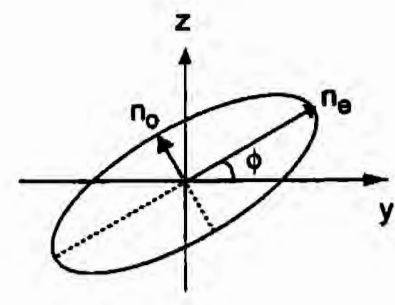

(a)

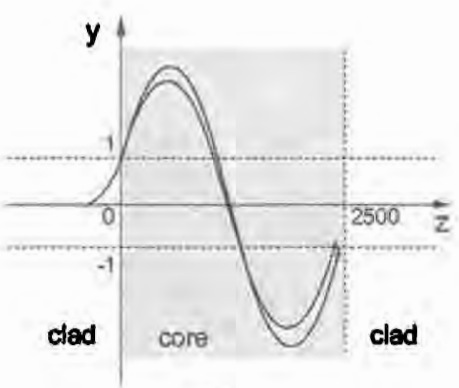

(b)

Figure 4. (a) Definition of the tilt angle $f$ of NLC molecule. (b) Schematic representation of shooting method for waveguide mode analysis.

\subsection{Numerical analysis}

In the presence of an applied electric field, NLC molecules reorient toward the direction of applied field and splay-bend deformation of NLC director is induced in the incident plane of two laser beams. In such a situation, only TM-guided mode is affected by the deformation of NLC director. Moreover, NLC waveguide should be treated as a graded index waveguide under an applied electric field, because the field-induced deformation of NLC molecules is inhomogeneous in depth, that is, NLC molecules in the vicinity of the substrates are anchored more tightly, and NLC molecules far from substrates can reorient more freely. Santamato et. al. reported the numerical analysis of TM modes in a slab waveguide filled with NLC in an external magnetic field ${ }^{20}$ and Lin et. $a .^{21}$ and Abbate et. al. ${ }^{22}$ have reported the numerical analysis of nonlinear effects in NLC waveguide. We have adopted their method for the calculation. The scheme of numerical analysis can be summarized as follows.

1. director profile in depth $\psi(z, V)$ upon an electric field should be solved based on the elastic theory.

2. optical anisotropy in the NLC core layer should be included in the Maxwell's equation as a tensor $\epsilon(z, V)$, and the propagation constant $\beta(V)$ (or effective refractive index $N(V)$ ) and the optical field profile of the guided mode should be calculated.

From the Oseen-Frank's elastic theory, the density of free energy $g(V)$ in the presence of applied electric field can be expressed as follows,

$$
g(V)=\frac{1}{2} K_{11}(\nabla \cdot n)^{2}+\frac{1}{2} K_{33}[n \times(\nabla \times n)]^{2}-\frac{1}{2} \epsilon_{0} \epsilon_{a}(E \cdot n)^{2}
$$

where $K_{11}$ and $K_{33}$ are splay and bend Frank's elastic constants, respectively, and $\epsilon_{a}\left(=\epsilon_{e}-\epsilon_{o}\right)$ is the dielectric anisotropy, and $\epsilon_{e}$ and $\epsilon_{o}$ are dielectric constant of NLC parallel and perpendicular to the director, respectively. Using director tilt angle $\psi(z, V)$ which is defined in Fig.4 (a), NLC director $n(z, V)$ can be expressed as $n(z, V)$ $=\left(n_{x}(z, V), n_{y}(z, V), n_{z}(z, V)\right)=(0, \cos \psi(z, V), \sin \psi(z, V))$, and following equation can be derived from minimization of free energy $g(V)$ assuming that NLC on substrates cannot move,

$$
\frac{d \psi(z, V)}{d z}=\sqrt{\frac{\epsilon_{0} \epsilon_{a} E^{2}}{K_{11}}} \sqrt{\frac{\sin ^{2} \psi_{m}(V)-\sin ^{2} \psi(z, V)}{\cos ^{2} \psi(z, V)+\left(K_{33} / K_{11}\right) \sin ^{2} \psi(z, V)}}
$$

where $\psi_{m}(V)$ is tilt angle of NLC molecules at the center of the cell (maximum tilt angle), and obtained as a solution of following integral. ${ }^{23}$

$$
\frac{E}{E_{c}}=\frac{2}{\pi} \int_{0}^{\psi_{m}} \sqrt{\frac{1+\left(K_{33} / K_{11}-1\right) \sin ^{2} \psi}{\sin ^{2} \psi_{m}-\sin ^{2} \psi}} d \psi
$$


The dielectric tensor $\epsilon(z, V)$ can be expressed using tilt angle $\psi(z, V)$ calculated above as follows,

$$
\begin{aligned}
\epsilon(z, V) & =\left[\begin{array}{ccc}
\epsilon_{\infty 11}(z, V) & \epsilon_{\infty 12}(z, V) & \epsilon_{\infty 13}(z, V) \\
\epsilon_{\infty 21}(z, V) & \epsilon_{\infty 22}(z, V) & \epsilon_{\infty 23}(z, V) \\
\epsilon_{\infty 31}(z, V) & \epsilon_{\infty 32}(z, V) & \epsilon_{\infty 33}(z, V)
\end{array}\right] \\
& =\left[\begin{array}{ccc}
\epsilon_{\infty o} & 0 & 0 \\
0 & \epsilon_{\infty o}+\epsilon_{\infty a} \cos ^{2} \psi(z, V) & \epsilon_{\infty a} \cos \psi(z, V) \sin \psi(z, V) \\
0 & \epsilon_{\infty a} \cos \psi(z, V) \sin \psi(z, V) & \epsilon_{\infty 0}+\epsilon_{\infty a} \sin ^{2} \psi(z, V)
\end{array}\right]
\end{aligned}
$$

where $\epsilon_{\infty a}\left(=\epsilon_{\infty e}-\epsilon_{\infty o}\right)$ is the dielectric anisotropy, and $\epsilon_{\infty e}\left(=n_{e}^{2}\right)$ and $\epsilon_{\infty o}\left(=n_{o}^{2}\right)$ are dielectric constant of NLC parallel and perpendicular to the director at optical frequency, respectively. TM-polarized light is defined as $\boldsymbol{H}=\left(H_{x}, 0,0\right)$ and $\boldsymbol{E}=\left(0, E_{y}, E_{z}\right)$, and by assuming monochromatic light

$$
H_{x}(y, z, t, V)=R(z, V) \exp [\psi(z, V)] \exp \left[i\left(k_{0} N(V) y-\omega t\right)\right]
$$

following equation can be obtained from Maxwell's equations.

$$
\epsilon_{\infty 33}(z, V)^{2} \frac{d^{2} R(z, V)}{d z^{2}}+\epsilon_{\infty 33}(z, V) \frac{d \epsilon_{\infty 33}(z, V)}{d z} \frac{d R(z, V)}{d z}+k_{0}^{2} \epsilon_{\infty \text { o }} \epsilon_{\infty e}\left(\epsilon_{\infty 33}(z, V)-N(V)^{2}\right) R(z, V)=0
$$

where $k_{0}$ is a wavenumber in vacuum, $\omega$ is frequency of guided light and $N(V)$ is an effective refractive index, which is defined using propagation constant $\beta(V)$ as $N(V)=\beta(V) / k_{0}$. This equation is second-order ordinary differential equation and can be regarded as an eigenvalue problem with the effective refractive index $N(V)$ playing the role of the eigenvalue, and cannot be solved analytically. In order to solve this problem, we have adopted shooting method employing $N(V)$ as a parameter (Fig.4 (b)). ${ }^{24}$ By solving equation (7), effective refractive index $N(V)$ and optical field profile $R(z, V)$ of the guided mode can be obtained.

$$
H_{x}(y, z, t, V)= \begin{cases}\exp \left[-k_{0} \sqrt{\beta(V)^{2}-n_{c}^{2}} z\right] & , z<0 \\ R(z, V) & , 0 \leq z \leq 2500 \mathrm{~nm} \\ ( \pm)^{m} \exp \left[-k_{0} \sqrt{\beta(V)^{2}-n_{c}^{2}}(z-2500)\right] & , z>2500 \mathrm{~nm}\end{cases}
$$

As sample parameters of the numerical calculation, typical values of widely used cyanobiphenyl based NLC mixture E7 (Merck) were used. Parameters used for numerical calculation are summerized in Table. 1.

Table 1. parameters used for the numerical calculation

\begin{tabular}{|l|c|c|}
\hline \hline & Symbols & Values \\
\hline ordinary refractive index of NLC & $n_{o}$ & 1.53 \\
extraordinary refractive index of NLC & $n_{e}$ & 1.78 \\
refractive index of PVA & $n_{P V A}$ & 1.52 \\
Frank's elastic constants (splay) & $K_{11}$ & $1.1 \times 10^{-11} \mathrm{~N}$ \\
Frank's elastic constants (bend) & $K_{33}$ & $1.7 \times 10^{-11} \mathrm{~N}$ \\
dielectric constant of NLC (parallel to the director) & $\epsilon_{e}$ & 19.0 \\
dielectric constant of NLC (perpendicular to the director) & $\epsilon_{o}$ & 5.7 \\
cell gap & $\mathrm{d}$ & $2.5 \mu \mathrm{m}$ \\
\hline \hline
\end{tabular}




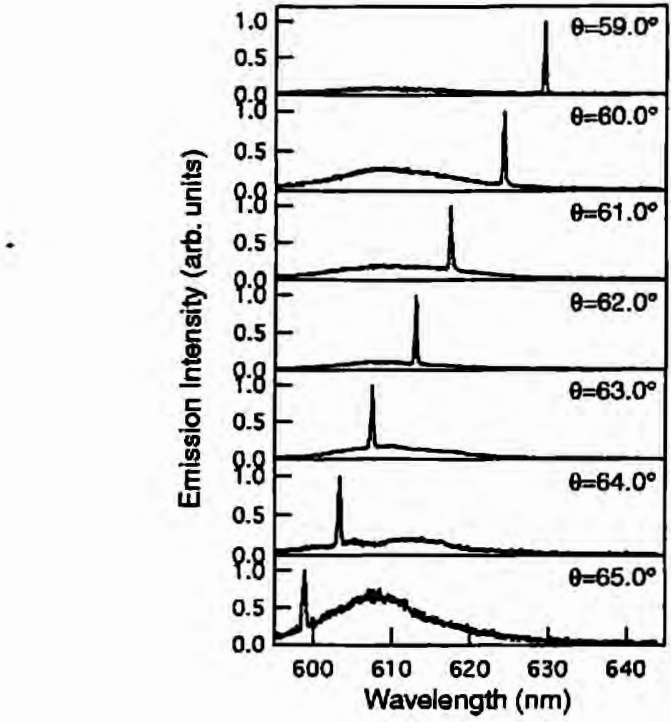

(a)

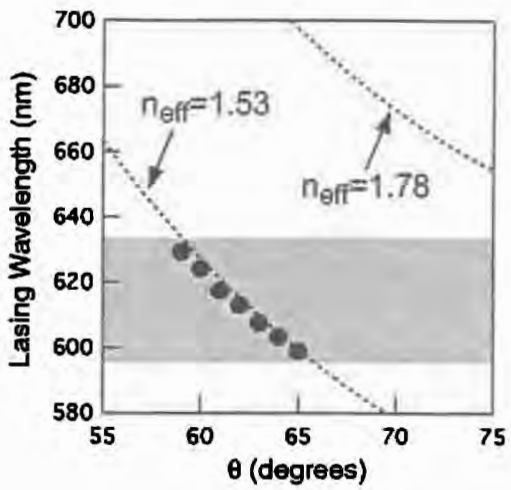

(b)

Figure 5. (a) Emission spectra of the dye-doped NLC waveguide upon holographic excitation at various excitation angles (from $59.0^{\circ}$ to $65.0^{\circ}$ by $1.0^{\circ}$ ) above the threshold pump pulse energy without applied voltage. (b) Excitation angle dependence of the lasing wavelength. The shaded region shows the tunable range of this laser. The dashed lines show the theoretical curves of the lasing wavelength under the first order diffraction condition $(m=1)$ assuming the effective refractive indices $n_{\text {ef } f}$ as 1.53 and 1.78 .

\section{RESULTS AND DISCUSSION}

\subsection{Experimental results}

Figure 5 (a) shows the emission spectra of the dye-doped NLC waveguide for various excitation angles (from $59.0^{\circ}$ to $65.0^{\circ}$ by $1.0^{\circ}$ ) above the threshold pump pulse energy without applied voltage. As is evident from the figure, the lasing wavelength could be tuned by changing $\theta$. The tunable range of lasing wavelength upon holographic excitation was $\sim 30 \mathrm{~nm}$, which was determined by the doped laser dye. Figure 5 (b) shows the excitation angle dependence of the lasing wavelength. The shaded region shows the tunable range of this laser. The dashed lines show the theoretical curves of the lasing wavelength under the first order diffraction condition $(m=1)$ assuming the effective refractive indices $n_{\text {eff }}$ as 1.53 and 1.78. The excitation angle dependence of the lasing wavelength agrees well with the theoretical curve with the effective refractive index $n_{\text {eff } f}$ of 1.53 . It was confirmed that the laser emission wavelength could be tuned by changing the excitation angle and that the DFB laser action was realized due to the holographically induced transient grating.

Figure 6 (a) shows the emission spectra of the dye-doped NLC waveguide at various applied voltages. The angle $\theta$ was fixed to be $65.0^{\circ}$. When the applied voltage was below $0.7 \mathrm{~V}$, laser emission peak was observed at $599 \mathrm{~nm}$ (peak 1) and no change was observed in the laser emission. When the applied voltage exceeded $\sim 0.8$ $\mathrm{V}$, another new peak (peak 2) appeared in longer wavelength. The peak 2 showed a continuous red-shift with increasing the applied voltage. Above the applied voltage of $\sim 1.0 \mathrm{~V}$, laser emission of peak 2 became multi-mode (peak 2 refer to all peaks which show red-shift with applied voltage in the spectrum). When applied voltage was more than $\sim 1.4 \mathrm{~V}$, laser emission disappeared. On the other hand, the peak 1 did not shift at all. The amplitude of the peak 1 decreased gradually, and finally disappeared above $1.1 \mathrm{~V}$. In Fig.6 (b), applied voltage dependences of the lasing wavelength of the peak 1 (open circle) and the peak 2 (closed circle) are summarized. The threshold voltage for the appearance of the peak 2 is observed at $\sim 0.8 \mathrm{~V}$, which should be attributed to the Frederiks transition of the NLC. The electrical tuning of laser action could be performed reversibly. The lasing wavelength is quite sensitive to the applied voltage, and the tuning of the lasing wavelength was realized slightly above the threshold voltage. 


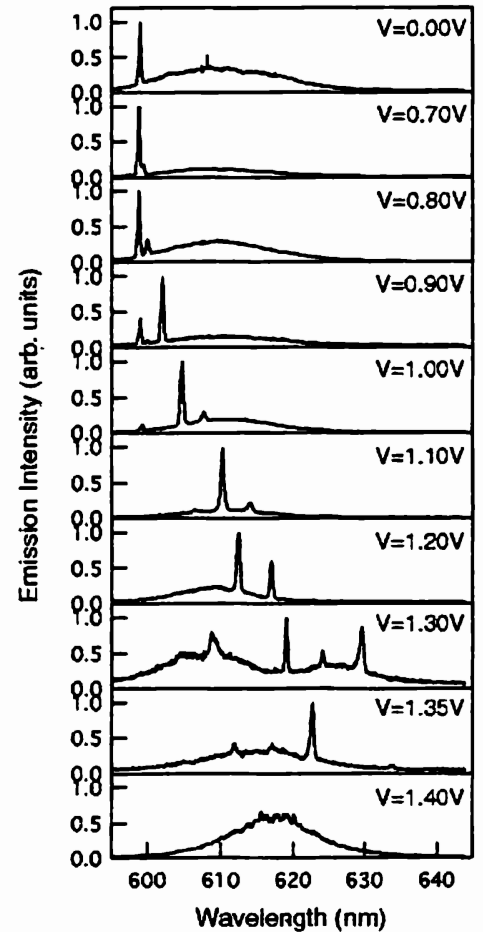

(a)

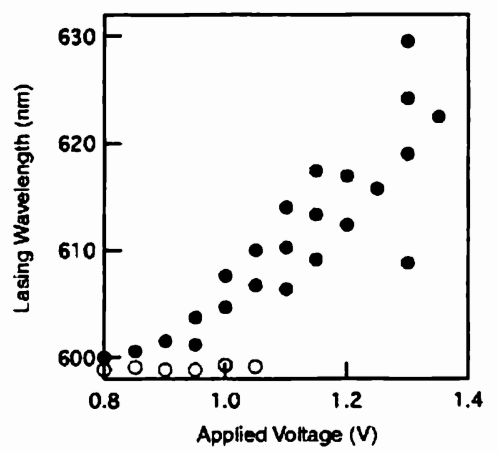

(b)

Figure 6. (a) Emission spectra of the dye-doped NLC waveguide upon holographic excitation at various applied voltages $\left(\theta=65.0^{\circ}\right)$. (b) Applied voltage dependence of lasing wavelength.

In order to explain these results, TE- and TM-guided modes should be treated separately. ${ }^{14}$ The reorientation of NLC molecules upon the application of an electric field takes place in the incident plane of two excitation beams, so that TE-guided mode feels $n_{o}$ of the NLC regardless of the applied voltage, and no change in lasing wavelength of the peak 1 should be attributed to the TE-guided mode. On the other hand, the effective refractive index $n_{\text {eff }}$ for the TM-guided mode increases gradually with the field-induced reorientation of the NLC molecules: and the lasing wavelength associated with TM-guided mode should shift to longer wavelength. Consequently; the red-shift of the lasing peak 2 might be attributed to the TM-guided mode. With the increase of the effective refractive index $n_{\text {eff } f}$, the effective thickness of the core layer also increases and higher order guided mode should appear. The appearance of multi-mode emission in peak 2 upon application of more than about $1.0 \mathrm{~V}$ should be explained in terms of this higher order guided mode. In order to analyze these results in detail, numerical analysis of TM-guided mode was performed as followings.

\subsection{Numerical analysis of TM-guided mode}

As a first step, distribution of the tilt angle $\psi(z, V)$ of NLC molecules upon applied voltages was calculated. Figure 7 (a) shows the calculated tilt angle of NLC molecules with various applied voltages $(V=1.00,1.50$, $2.00,4.00,6.00 \mathrm{~V})$. Using these data $\psi(z, V)$, the TM-guided mode with applied voltages was calculated.

Figure 7 (b) shows the applied voltage dependence of the effective refractive index $N(V)$ of the 0th $(m=$ 0 ) ordered guided mode in the waveguide core layer. The wavelength of the guided light was set to be $600 \mathrm{~nm}$. It can be seen that the change of $N(V)$ is steep just above the threshold applied voltage for the reorientation of NLC molecules; and saturates when applied voltage is larger than about $2.0 \mathrm{~V}$. Following discussions focus mainly on the applied voltage range slightly above the threshold.

Figure 8 (a) shows the applied voltage dependence of $N(V)$ in the waveguide core laver, which are for TMguided modes with monochromatic lights of $\lambda=600 \mathrm{~nm}$ (circles) and $\lambda=630 \mathrm{~nm}$ (triangles). When applied 


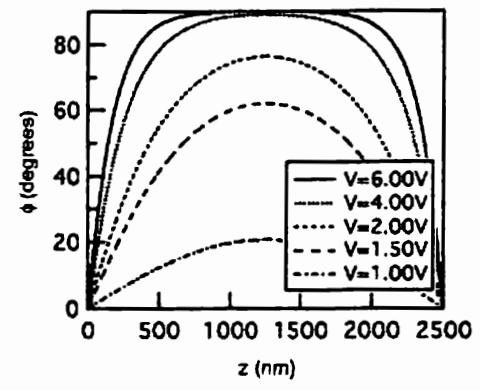

(a)

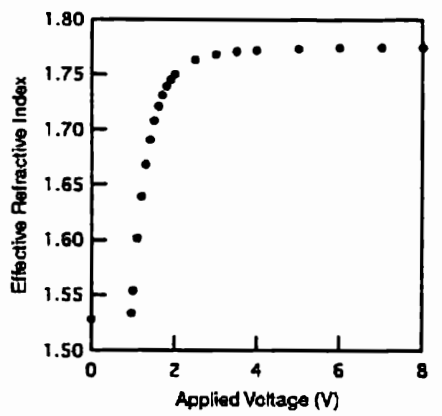

(b)

Figure 7. (a) Distribution of tilt angle of NLC molecules in the depth of the cell under various applied voltages ( $\mathrm{V}=$ $1.00,1.50,2.00,4.00$ and $6.00 \mathrm{~V}$ ). (b) Applied voltage dependence of effective refractive index of 0th ordered TM-guided mode with the wavelength of $\lambda=600 \mathrm{~nm}$.

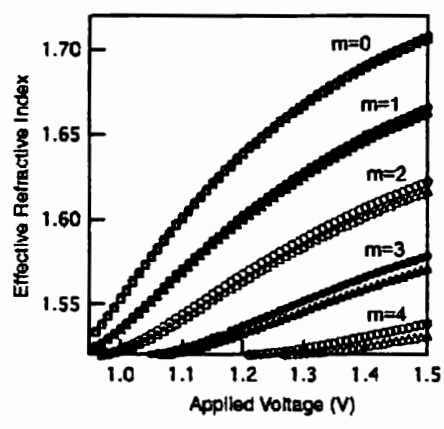

(a)

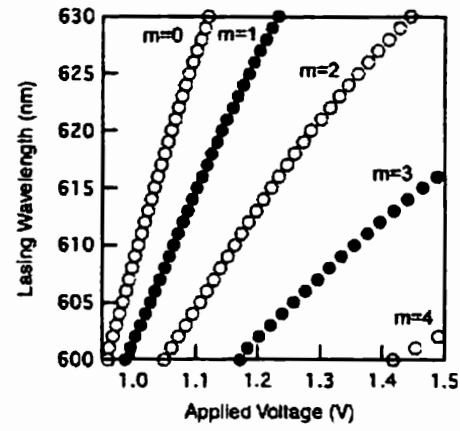

(b)

Figure 8. (a) Applied voltage dependence of effective refractive index of from 0 th to 4 th ordered TM-guided mode with the wavelength of $\lambda=600 \mathrm{~nm}$ (circles) and $\lambda=630 \mathrm{~nm}$ (triangles). (b) Applied voltage dependence of lasing wavelength of from 0th to 4th ordered TM-guided mode.

voltage was increased, $N(V)$ also increased and higher ordered guided modes appeared. As can be seen in Fig. 8 (a), if wavelength of the guided lights are different, $N(V)$ are also different. Consequently, it can be considered that for the calculation of lasing wavelength, different voltage dependence of $N(V)$ should be used for each wavelength. The calculation procedures of the applied voltage dependence of lasing wavelength are summarized as follows.

1. Calculate voltage dependence of $N(V)$ for each wavelength of the guided light same as in Fig. 8 (a).

2. From these results, pick up the applied voltages which agree with $N(V)$ which satisfy the lasing condition shown in equation (1) for each lasing wavelength. For example, in the case of $\lambda=630 \mathrm{~nm}$, $n_{\epsilon f f}$ for lasing is estimated as 1.608 (assuming $\theta$ as $65.0^{\circ}$ ) from equation (1) and applied voltage can be derived as $V=$ $1.119 \mathrm{~V}$ for 0 th ordered guided mode from Fig. 8 (a).

3. Plot the lasing wavelength versus applied voltages.

Figure 8 (b) shows the calculated voltage dependence of lasing wavelength. It can be seen that when applied voltage increased. lasing wavelength shifts toward longer wavelength. At high applied voltages. the lower ordered guided mode deviates from tunable range of lasing (in this case, from 600 to $630 \mathrm{~nm}$ ), and higher ordered guided 
(a) $V=0.00 \mathrm{~V}$

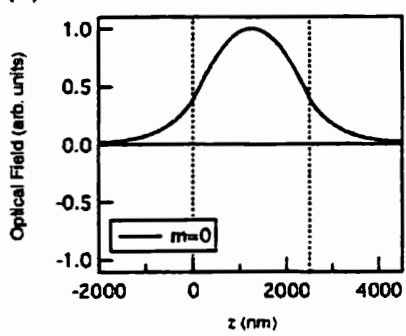

(d) $V=1.20 \mathrm{~V}$

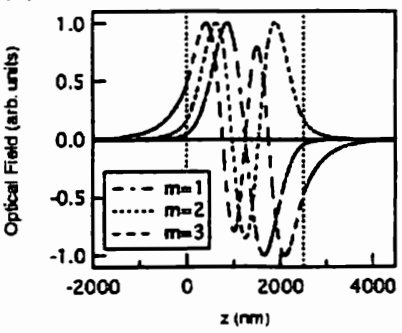

(b) $V=1.00 \mathrm{~V}$

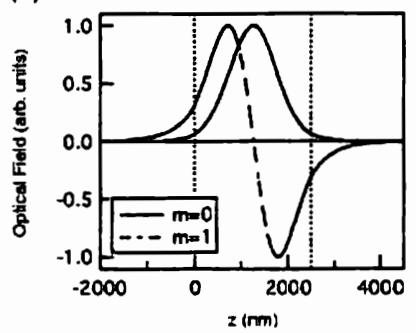

(e) $V=1.30 \mathrm{~V}$

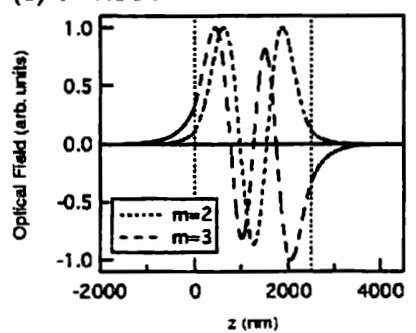

(c) $V=1.10 \mathrm{~V}$

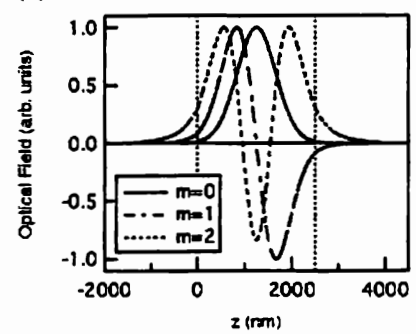

(f) $V=1.40 \mathrm{~V}$

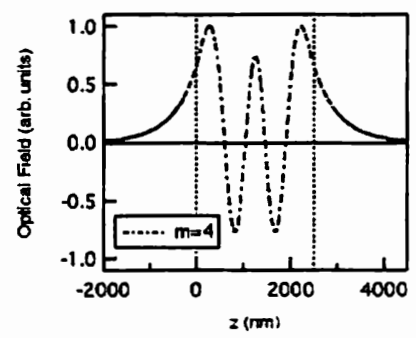

Figure 9. Optical field profile of TM-guided mode without (a) and with applied voltages ((b) $1.00 \mathrm{~V}$, (c) $1.10 \mathrm{~V}$, (d) $1.20 \mathrm{~V}$, (e) $1.30 \mathrm{~V}$, (f) $1.40 \mathrm{~V}$ ). Boundaries of the PVA clad layer ( $z<0$ and $z>2500$ nm) and the NLC core layer $(0<z<2500 \mathrm{~nm})$ are also shown in dotted lines. For the calculation, the wavelength of the guided light were set as follows: (a) $\lambda_{m=0}=600 \mathrm{~nm}$, (b) $\lambda_{m=0}=609 \mathrm{~nm}, \lambda_{m=1}=601 \mathrm{~nm}$. (c) $\lambda_{m=0}=627 \mathrm{~nm}, \lambda_{m=1}=615 \mathrm{~nm}, \lambda_{m=2}=604$ $\mathrm{nm}$, (d) $\lambda_{m=1}=627 \mathrm{~nm}, \lambda_{m=2}=613 \mathrm{~nm}, \lambda_{m=3}=602 \mathrm{~nm},(\mathrm{e}) \lambda_{m=2}=621 \mathrm{~nm}, \lambda_{m=3}=607 \mathrm{~nm}$, (f) $\lambda_{m=4}=600 \mathrm{~nm}$.

modes appear. As easily estimated from these results: more higher ordered guided modes should even appear at more higher applied voltages. If all higher-ordered guided modes can contribute to lasing, experimentally observed disappearance of lasing at high voltages cannot be explained. From this estimation, the efficiency of the confinement of the guided light in the waveguide core layer is considered to play a crucial role for lasing. In order to analyze this effect, optical field profiles of guided mode upon applied voltages were calculated.

Figure 9 shows the optical field profile of TM-guided mode without (a) and with applied voltages ((b) 1.00 $\mathrm{V}$ : (c) $1.10 \mathrm{~V}$. (d) $1.20 \mathrm{~V}$, (e) $1.30 \mathrm{~V}$, (f) $1.40 \mathrm{~V}$ ). Boundaries of the PVA clad layer $(z<0$ and $z>2500 \mathrm{~nm}$ ) and the NLC core layer $(0<z<2500 \mathrm{~nm})$ are also shown in dotted lines. For the calculation, the wavelength of the guided light were set as follows: (a) $\lambda_{m=0}=600 \mathrm{~nm}$, (b) $\lambda_{m=0}=609 \mathrm{~nm}, \lambda_{m=1}=601 \mathrm{~nm}$, (c) $\lambda_{m=0}=$ $627 \mathrm{~nm}, \lambda_{m=1}=615 \mathrm{~nm}, \lambda_{m=2}=604 \mathrm{~nm},(\mathrm{~d}) \lambda_{m=1}=627 \mathrm{~nm}, \lambda_{m=2}=613 \mathrm{~nm}, \lambda_{m=3}=602 \mathrm{~nm},(\mathrm{e}) \lambda_{m=2}$ $=621 \mathrm{~nm}, \lambda_{m=3}=607 \mathrm{~nm}$, (f) $\lambda_{m=4}=600 \mathrm{~nm}$. These values were chosen in order to agree the values of the calculated lasing wavelength shown in Fig.8 (b). Without or with a low applied voltages slightly above the threshold applied voltage, optical fields are confined well in the waveguide core layer as shown in Figs.9 (a)-(e). On the other hand, with high applied voltage $(>1.40 \mathrm{~V})$, confinement of optical fields in the waveguide core layer cannot be carried out effectively as shown in Fig.9 (f). As easily estimated from these results, although more higher ordered guided modes should appear even at higher applied voltages from the result shown in Fig.8, efficiency of confinement in the waveguide core layer will not be high. As a result, the reason why no lasing was observed at higher applied voltages can be attributed to the low efficiency of confinement of higher ordered guided modes.

\subsection{Prospect for realization of single mode operation}

For the device application, a single-mode operation is more desirable. For the realization of the single mode operation, the waveguide should be designed to confine only the lowest (0th) ordered guided mode effectively. Since the number of guided-mode depends on the effective thickness of the core layer, possible approaches for the realization of single-mode operation can be summarized as follows. 
(a) $\mathrm{V}=0.00 \mathrm{~V}$

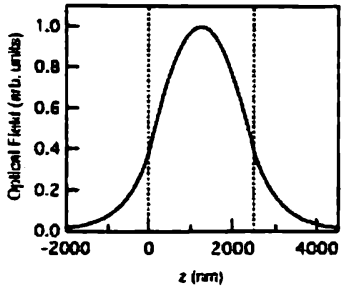

(d) $V=2.00 \mathrm{~V}$

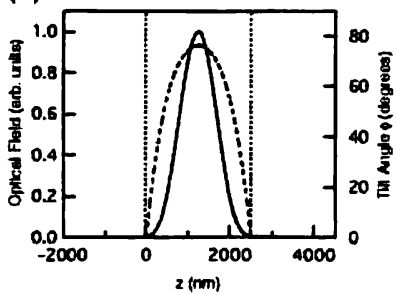

(b) $\mathrm{V}=1.00 \mathrm{~V}$

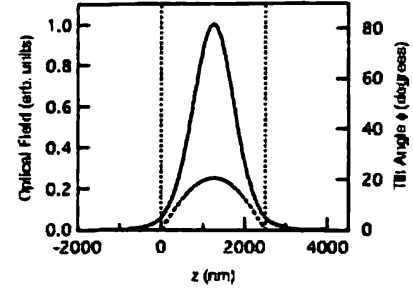

(e) $\mathrm{V}=4.00 \mathrm{~V}$

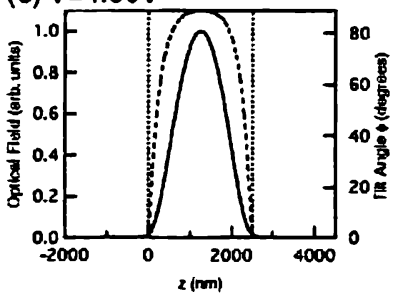

(c) $\mathrm{V}=1.50 \mathrm{~V}$

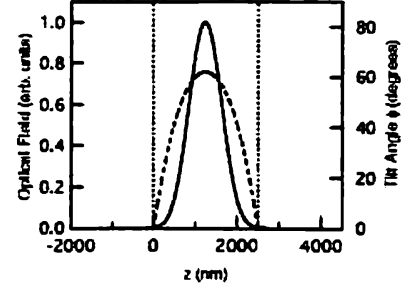

(f) $\mathrm{V}=6.00 \mathrm{~V}$

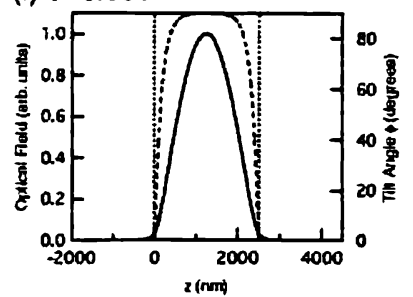

Figure 10. Optical field profile of Oth ordered TM-guided mode without (a) and with applied voltages (b-f). Wavelength of the guided light $\lambda=600 \mathrm{~nm}$. The distribution of the tilt angle $\psi(z)$ in depth are also shown in dotted lines for reference.

1. Make an effective thickness of waveguide core layer thinner. This includes physical thickness itself, and low index contrast between core and clad layers.

2. Utilize another configuration of LC cell, such as hybrid aligned nematic (HAN) cell, ${ }^{25} \pi$-cell, ${ }^{26}$ and so on. Using these inhomogeneous director profiles, in which the core layer can be regarded as the graded index waveguide, confine electromagnetic field in thin region (only middle part) in the cell effectively.

In order to estimate which approach is more significant for the single-mode lasing, optical field profiles of 0th ordered TM-guided mode were analyzed. From this result, the importance of the second model (graded profile of tilt angle) can be evaluated, because the distribution of the tilt angle of NLC upon high electric field application in the cell we employed is similar to that of $\pi$-cell.

Figure 10 shows the numerically analyzed optical field profile of 0th ordered TM-guided mode without (a) and with applied voltages (b-f). Wavelength of guided light $\lambda=600 \mathrm{~nm}$. The distribution of the tilt angle $\psi(z, V)$ in depth are also shown in dotted lines for reference. With sufficiently high voltages, homeotropic region of NLC grows larger at the center of the core layer and spread all over the core layer, and effective thickness of the core layer glows thicker. This means that for the effective confinement of the guided mode, there is an optimal range of applied voltage. From Fig.10, this optimal applied voltage seems to lie around $\sim 1.5 \mathrm{~V}$. As a result, first approach, that is, to lower $\Delta n\left(=n_{e}-n_{o}\right)$ or $n_{\varepsilon}$ seem to be more important.

\section{4. single-mode operation of lasing}

Based on these considerations, a lower-index NLC was used. ${ }^{27}$ As a NLC host. ZLI1132 (Merck) with $n_{e}$ and $n_{0}$ of 1.63 and 1.50, respectively was used. The dielectric anisotropy of this NLC is also positive. The concentration of the dye. DCM, was $0.6 \mathrm{wt} . \%$. The cell gap was $2.2 \mu \mathrm{m}$.

Figure 11 (a) shows the emission spectra at various applied voltages. The excitation angle $\theta$ was maintained at $64.0^{\circ}$. At this angle, the laser action was observed at the longer wavelength edge of the photoluminescence region of the doped dye $(\lambda=641.1 \mathrm{~nm})$ with the application of $6.0 \mathrm{~V}$. From $6.0 \mathrm{~V}$, the applied voltage was reduced gradually. Continuous blueshift of the lasing wavelength was observed. The lasing peak disappeared below approximately $2.0 \mathrm{~V}$. Because the guided mode can no longer be confined in such a low voltage, lasing may not be observed. Figure 11 (b) shows the applied voltage dependence of the lasing wavelength. It can be 


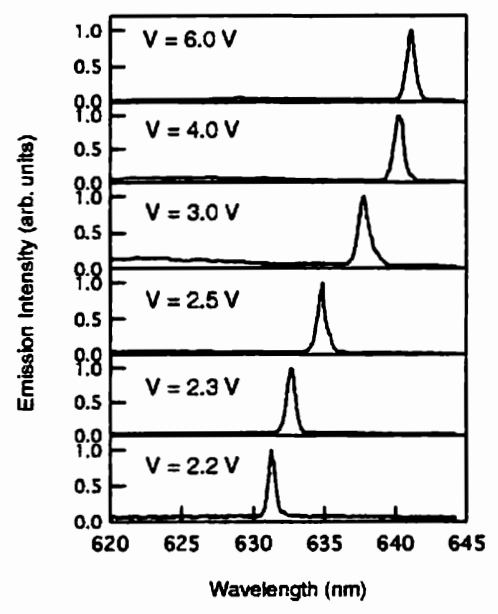

(a)

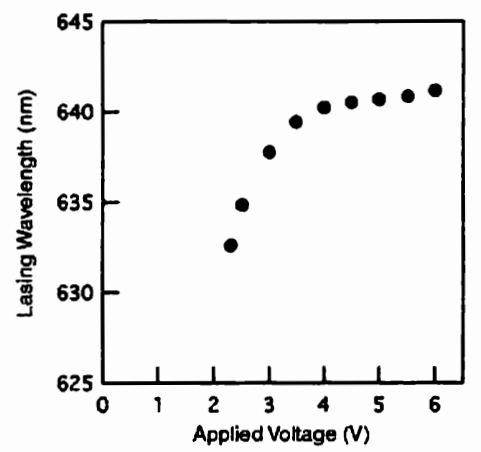

(b)

Figure 11. (a) Emission spectra of the dye-doped NLC waveguide upon holographic excitation at various applied voltages $\left(\theta=64.0^{\circ}\right)$. (b) Applied voltage dependence of lasing wavelength $\left(\theta=64.0^{\circ}\right)$.

seen that the field-induced shift of the lasing wavelength is continuous, and particularly marked from 2.0 to 3.0 $V$. The total shift of the lasing wavelength is about $8.5 \mathrm{~nm}$.

Although the single-mode operation of lasing was realized, a detailed mechanism is not clear at this stage. Moreover, there are still problems that should be solved, namely, high operation voltage and narrow tunable range of the lasing wavelength. For the design of a more efficient device structure, further optimization of these parameters is required.

\section{CONCLUSIONS}

In conclusion, optically pumped DFB laser action was observed in the dye-doped NLC waveguide. The DFB laser action was realized by the holographic excitation using the Lloyd mirror setup. Upon an applied electric field, the wavelength of the laser emission was continuously tuned on the basis of the reorientation of NLC molecules. TM-guided modes under applied voltages were analyzed numerically based on the waveguiding mode theory, and the agreement with the experimental results was demonstrated. Moreover, single-mode operation of lasing was also demonstrated. Our device seems to be more complicated than the cases employing the laser dye solution as an active medium. The origin of this complication can be attributed to waveguide structure, inhomogeneous distribution of tilt angle of NLC molecules $\psi(z, V)$ under applied voltages, anisotropy of refractive indices of NLC molecules, and so on. Using these parameters effectively, various tunable lasers can be designed based on the waveguiding mode theory.

\section{ACKNOWLEDGMENTS}

This work is in part supported by a Grant-in-Aid for Scientific Research from the Japan Ministry of Education, Culture, Sports, Science and Technology. T.M. acknowledges financial support from Grant-in-Aid for Japan Society for the Promotion of Science (JSPS) Fellows.

\section{REFERENCES}

1. H. Kogelnik and C. V. Shank, "Stimulated emission in a periodic structure," Appl. Phys. Lett. 18, pp. 152-154, 1971.

2. A. Dodabalapur, E. A. Chandross, M. Berggren and R. E. Slusher, "Organic solid-state lasers: past and future," Science 277, pp. 1787-1788, 1997. 
3. V. I. Kopp. B. Fan, H. K. Vithana. A. Z. Genack, "Low-threshold lasing at the edge of a photonic stop band in cholesteric liquid crystals," Opt. Lett. 23, pp. 1707-1709. 1998.

4. B. Taheri, A. F. Munoz. P. Palffy-Muhoray and R. Twieg, "Low threshold lasing in cholesteric liquid crystals," Mol. Cryst. Liq. Cryst. 358, pp. 73-82, 2001.

5. H. Finkelmann, S. T. Kim, A. Munoz, P. Palffy-Muhoray, B. Taheri. "Tunable mirrorless lasing in cholesteric liquid crystalline elastomers." Adv. Mater. 13, pp. 1069-1072, 2001.

6. J. Schmidtke, W. Stille, H. Finkelmann and S. T. Kim. "Laser emission in a dye doped cholesteric polymer network:" Adv. Mater. 14. pp. 746-749. 2002.

7. T. Matsui, R. Ozaki, K. Funamoto, M. Ozaki and K. Yoshino, "Flexible mirrorless laser based on a freestanding film of photopolymerized cholesteric liquid crystal," Appl. Phys. Lett. 81, pp. 3741-3743, 2002.

8. M. Ozaki, M. Kasano, D. Ganzke, W. Haase and K. Yoshino, "Mirrorless lasing in a dye-doped ferroelectric liquid crystal," Adv. Mater. 14, pp. 306-309, 2002.

9. M. Ozaki, M. Kasano, T. Kitasho, D. Ganzke, W. Haase and K. Yoshino, "Electro-tunable liquid-crystal laser," Adv. Mater. 15, pp. 974-977, 2003.

10. M. Berggren, A. Dodabalapur, R. E. Slusher, A. Timko and O. Nalamasu, "Organic solid-state lasers with imprinted gratings on plastic substrates," Appl. Phys. Lett. 72, pp. 410-411. 1998.

11. T. Matsui, M. Ozaki, K. Yoshino and F. Kajzar, "Fabrication of flexible distributed feedback laser using photoinduced surface relief grating on azo-polymer film as a template," Jpn. J. Appl. Phys. 41, pp. L1386L1388, 2002.

12. P. G. de Gennes, J. Prost, The Physics of Liquid Crystals, Oxford Univ. Press, New York, 1993.

13. R. Ozaki, T. Matsui, M. Ozaki and K. Yoshino, "Electrically color-tunable defect mode lasing in onedimensional photonic-band-gap system containing liquid crystal." Appl. Phys. Lett. 82, pp. 3593-3595, 2003.

14. T. Matsui, M. Ozaki and K. Yoshino, "Electro-tunable laser action in a dye-doped nematic liquid crystal waveguide under holographic excitation," Appl. Phys. Lett. 83. pp. 422-424, 2003.

15. C. V. Shank, J. E. Bjorkholm and H. Kogelnik, "Tunable distributed-feedback dye laser," Appl. Phys. Lett. 18. pp. 395-396. 1971.

16. D. J. Channin, "Optical waveguide modulation using nematic liquid crystal," Appl. Phys. Lett. 22, pp. 365-366, 1973.

17. J. P. Sheridan and T. G. Giallorenzi, "Electro-optically induced deflection in liquid-crystal waveguides," J. Appl. Phys. 45, pp. 5160-5163, 1974.

18. H. J. Eichler. P. Gunter and D. W. Pohl, Laser Induced Dynamic Gratings, Springer-Verlag, Berlin, 1985.

19. N. K. Viswanathan, S. Balasubramanian, L. Li, S. K. Tripathy and J. Kumar, "A detailed investigation of the polarization-dependent surface-relief-grating formation process on azo polymer films," Jpn. J. Appl. Phys. 38, pp. 5928-5937, 1999.

20. E. Santamato, P. Maddalena, M. Settembre, M. Romagnoli and B. Daino, "TM modes in a slab waveguide filled with nematic liquid crystal in an external magnetic field," J. Opt. Soc. Am. B 6, pp. 126-130, 1989.

21. H. Lin and P. Palffy-Muhoray, "Propagation of TM modes in a nonlinear liquid-crystal waveguide," Opt. Lett. 19, pp. 436-438, 1994.

22. G. Abbate, L. De Stefano and E. Santamato, "Transverse-magnetic nonlinear modes in a nematic liquidcrystal slab waveguide." J. Opt. Soc. Am. B 13. pp. 1536-1541, 1996.

23. H. Gruler and G. Meier, "Electric field-induced deformations in oriented liquid crystals of the nematic type," Mol. Cryst. Liq. Cryst. 16, pp. 299-310. 1972.

24. W. H. Press, B. P. Flannery, S. A. Teukolosky and W. T. Vetterling, Numerical Recipes in C, Cambridge Univ. Press, Cambridge, 1988.

25. M. Marangoni. R. Osellame, R. Ramponi, M. Buscaglia. T. Bellini and F. Mantegazza: "Field-controlled optical profile of a waveguide having a liquid-crystalline core:" Appl. Phys. Lett. 81, pp. 2337-2339. 2002.

26. P. J. Bos, P. Johnson and R. Koehler, "A liquid-crystal optical-switching device ( $\pi$ cell)." SID 83 Digest. pp. 30-31, 1983.

27. T. Matsui, M. Ozaki and K. Yoshino, "Single-mode operation of electrotunable laser in a dye-doped nematic liquid-crystal waveguide under holographic excitation." Jpn. J. Appl. Phys. 42, pp. L1462-L1464. 2003. 